\title{
Diferencias de los estilos de enseñanza: Mando directo y Resolución de problemas en el resultado para la enseñanza de la cualidad física de fuerza de tren inferior
}

\author{
Differences in the direct command teaching style and problem resolution in the \\ teaching result of the physical quality of lower body strength \\ *José Andrés Duarte Cornejo
}

Duarte, J. (2017). Diferencias de los estilos de enseñanza: Mando directo y Resolución de problemas en el resultado para la enseñanza de la cualidad física de fuerza de tren inferior. Revista Ciencias de la Actividad Física UCM, N $18(2)$ juliodiciembre, 1-10.

\begin{abstract}
RESUMEN
Se debe considerar al docente como aquel profesional reflexivo, que realiza una labor de mediación entre el conocimiento y el aprendizaje de sus estudiantes. ¿Qué estilo de enseñanza es más apropiado para mejorar el rendimiento de los estudiantes?, preguntas como éstas son las que se deben dar respuestas a la hora de realizar una planificación de la clase. El objetivo fue comparar el estilo de enseñanza, Mando directo y Resolución de problemas, mediante clases realizadas utilizando ambas metodologías y su influencia en la fuerza del tren inferior. Fue realizado en un primero medio bajo un método cuasi-experimental y cuyos resultados arrojaron que existen diferencias estadísticamente significativas entre ambas metodologías ( $\mathrm{p}$-valor $=0,014$ ). En la muestra independiente de post-test de salto largo a pies juntos en comparación a ambos estilos de enseñanza, se presentan diferencias estadísticamente significativas ( $\mathrm{p}$-valor $=0,046)$, a un nivel de confianza del 95\% $(\mathrm{p}<0,05)$. Una característica relevante de esta investigación, es la utilización de los estilos de enseñanza por parte de los docentes.
\end{abstract}

\section{PALABRAS CLAVE}

Estudiantes, estilos de enseñanza, Mando directo, Resolución de problemas.

\begin{abstract}
The teacher should be considered as that reflective professional, who mediates between the knowledge and learning of his students. What teaching style is most appropriate to improve student performance? Questions such as these are the ones that must be answered when planning the class. The objective was to compare the style of teaching, direct command and problem solving, through classes made using both methodologies and their influence on the strength of the lower train. It was carried out in a first medium under a quasi-experimental method and the results showed that there are statistically significant differences between both methodologies $(p$-value $=0,014)$. In the independent sample of post-test of long jump to feet together in comparison to both teaching styles, if statistically significant differences are presented ( $\mathrm{p}$-value $=0,046$ ), which gives us a statistically significant difference to a level of confidence $95 \%(p<0,05)$. One of the most relevant features of this research is the use of teaching styles by teachers.
\end{abstract}

\section{Key words}

Students, teaching styles, command style, problem solving.

* Facultad de Educación, Universidad Autónoma de Chile, Santiago, Chile. 


\section{INTRODUCCIÓN}

Hoy en día los docentes han sido bastante cuestionados tanto por sus conocimientos disciplinares como pedagógicos, quedando así en tela de juicio su formación y competencias. Un ejemplo concreto de esta situación es la incorporación que realizó el año 2008, elige educar, que presentó la evaluación inicia, como aquella evaluación diagnóstica, formativa y no habilitante centrada en conocimientos disciplinares y pedagógicos que se realiza a estudiantes egresados de párvulo y pedagogía, cuyo propósito es verificar la calidad de la formación inicial docente.

Según Ibarra (2006) señala que el rol del docente ha tenido múltiples cambios y su función está determinada por lo que la sociedad requiere en la actualidad. El docente pasó de ser el protagonista que entrega conocimientos (conductismo), a ser el mediador que facilita el aprendizaje a través de herramientas en donde, el alumno es partícipe en la construcción de estos conocimientos (Constructivismo).

Según Educarchile (2006), considera al docente como aquel profesional reflexivo, que realiza una labor de mediación entre el conocimiento y el aprendizaje de sus estudiantes, al compartir experiencias y saberes en un proceso de negociación o construcción conjunta del conocimiento y presta una ayuda pedagógica ajustada a la diversidad de necesidades, intereses y situaciones en que se involucran sus estudiantes ; es decir, la función central del docente es esencialmente orientar y guiar la actividad mental constructiva de sus estudiantes, a quienes proporcionará ayuda pedagógica ajustada a su competencia.

Es ahí donde nos surgen preguntas, que como docentes deberíamos resolver. Algunas preguntas que aborda esta investigación son: ¿todos los estudiantes aprenderán de la misma forma? ¿La metodología escogida, para los 40 o 45 estudiantes, llevará al éxito y no al fracaso? ¿Qué ocurre con los estudiantes que no entienden o no aprenden con la misma metodología? ¿Qué estilo de enseñanza es más apropiado para mejorar el rendimiento de los estudiantes? ¿Qué es más importante, los conocimientos del profesor o la metodología utilizada?;Generar buenos resultados depende de la metodología utilizada para el aprendizaje del alumno?

El presente estudio buscó analizar y comparar dos metodologías de enseñanza con el propósito de establecer cuál de ellas obtiene resultados más favorables dentro del proceso enseñanza- aprendizaje.

A raíz de ese propósito, se estableció como objetivo medular de la investigación, comparar el estilo de enseñanza, Mando directo y Resolución de problemas, mediante clases realizadas utilizando ambas metodologías, en el colegio Mistral de la comuna Las Cabras, para la enseñanza de la cualidad física de fuerza del tren inferior, en la prueba de salto largo a pies juntos.

Además se planteó una hipótesis de investigación, en la cual proyecta que existen cambios estadísticamente significativos entre el estilo de enseñanza alternativo (Resolución de problemas) y el estilo de enseñanza tradicional (Mando directo) en la cualidad física de fuerza del tren inferior aplicados a la prueba de salto largo a pies juntos, en el colegio Mistral de la comuna Las Cabras.

\section{METODOLOGÍA}

Este estudio es de tipo cuantitativo, de diseño cuasi-experimental de pre-test y post-test con un grupo control intacto, el cual estuvo compuesto por estudiantes de primero medio A del colegio Mistral, de la comuna Las Cabras. Se le aplicó una intervención con el estilo de enseñanza Resolución de problemas al grupo experimental. (Sampieri, 2006).

Según Delgado Noguera (1991), menciona los estilos de implicancia cognitiva, como aquellos que plantean situaciones de enseñanza que conlleva al alumno/a a buscar soluciones, a resolver problemas mediante la indagación e investigación, que intentan estimular un aprendizaje activo y significativo a través de la 
indagación y la experimentación motriz, lo que traslada la toma de decisiones al alumno.

El estilo de enseñanza de Resolución de problemas es un estilo representativo de la enseñanza por técnica de indagación, y se define como uno de los niveles más avanzados cognitivamente debido a que los ejercicios realizados poseen un gran número de respuestas que el alumno debe experimentar físicamente, lo esencial en este estilo es que el estudiante llegue a la respuesta del problema presentado lo que requiere una gran participación de él a nivel cognitivo como motor. Por su parte el docente debe estimular y retroalimentar ofreciendo sugerencias adicionales a las posibles respuestas para resolver el problema. (Echeverría, 2014).

La población objetivo del estudio estuvo sujeta a tanto niños como niñas con los siguientes criterios de inclusión: estudiantes de primer año medio del colegio particular subvencionado Mistral, de la comuna de Las Cabras, región de O’Higgins. Y que comprende a un total de 71 estudiantes. Los criterios de exclusión fueron delimitados por aquellos estudiantes que no asisten de forma regular o presentan algún impedimento físico.

\section{Tabla 1}

Distribución de estudiantes de primero medio según su sexo.

\begin{tabular}{lccc}
\hline \multicolumn{1}{c}{ Cursos } & Damas & Varones & $\begin{array}{c}\text { Total de } \\
\text { estudiantes }\end{array}$ \\
\hline $\begin{array}{l}\text { 1ro medio A } \\
\text { Mando directo }\end{array}$ & 20 & 18 & 38 \\
\hline $\begin{array}{l}\text { 1ro medio B } \\
\text { Resolución de } \\
\text { problemas }\end{array}$ & 15 & 18 & 33 \\
\hline Total & 35 & 36 & 71 \\
\hline
\end{tabular}

Se trabajo con la totalidad de la problación y al ser un diseño cuasi-experimental, se trabajó con los curso ya conformados. La determinación de los grupos control y experiemtnal se elaboró de manera probabilística utilizando el método de muestreo aleatorio simple (tómbola), se trabajó con la totalidad de la matrícula de cada curso. Independiente de pertenecer al colegio Mistral y al curso de primer año medio, se les solicitó la firma del consentimiento informado a cada uno de ellos.

La distribución de la muestra para esta investigación se dividió en dos grupos; control y experimental. Donde el grupo control trabajó con el estilo tradicional (Mando directo) y el grupo experimental con el estilo alternativo (Resolución de problemas).

En cuanto al plan de trabajo y antes de comenzar con la evaluación diagnóstica, se procedió a explicar el tipo de ejercicio a realizar por parte del profesor para evitar el desconocimiento del alumno frente a él, luego se procedió a aplicar el test de salto largo a pies juntos, validada por educar chile el año 2009, la cual es una evaluación que se encuentra dentro de las baterías de pruebas del SIMCE.

Posteriormente se aplicó un plan de entrenamiento de 6 semanas aproximadamente, conn una frecuencia de 2 veces a la semana (cada sesión de 2 horas pedagógicas) el cual buscó que el estudiante le diera una mayor respuesta o solución a problema establecido por el docente, cada planificación siguió la estructura planteada por los planes y progrmads establecidos por el gebierno, dando un énfasis al desarrollo cognitivo del estudiante. Posteriormente se realizó la toma de post-test del salto longitudinal, para observar si existen diferencias estadisticamente significativas entre ambos grupos que utilizaron modelos de enseñanza distintos.

\section{RESULTADOS}

Para el análisis de resultados, primero se realizó una prueba de normalidad, posteriormente, se utilizó estadígrafo t de student, para verificar la existencia de diferencias significativas entre las medias de una variable aplicando un pre-test y un post-test, así como un análisis de ANOVA de un factor. 
Tabla 2

Prueba de normalidad.

\begin{tabular}{lllll} 
& & \multicolumn{3}{c}{ Kolmogorov-Smirnov $^{\mathrm{a}}$} \\
\cline { 2 - 5 } Salto_pre & Estilo enseñanza & Estadístico & $\mathrm{gl}$ & Sig. \\
\cline { 2 - 5 } & Mando directo & 0,098 & 33 & $0,200^{*}$ \\
\cline { 2 - 5 } Salto_post & Resolución de problemas & 0,099 & 29 & $0,200^{*}$ \\
\cline { 2 - 5 } & Mando directo & 0,077 & 33 & $0,200^{*}$ \\
\hline
\end{tabular}

En la Tabla 2 se puede observar la prueba de normalidad de Kolmogorov-Smirnov, debido a que las observaciones son mayores a 50 casos. La población observada presentó una distribución normal, debido a que los valores obtenidos son mayores a p-valor $>0,05$ ( $\mathrm{p}$-valor $=0,200)$, donde no se encontraron diferencias significativas, lo que pudo concluir que la población presentó una distribución normal.

Tabla 3

Prueba de muestras relacionadas.

\begin{tabular}{|c|c|c|c|c|c|c|c|c|c|}
\hline & & \multicolumn{5}{|c|}{ Diferencias relacionadas } & \multirow[b]{3}{*}{$\mathrm{t}$} & \multirow[b]{3}{*}{$\mathrm{gl}$} & \multirow{3}{*}{$\begin{array}{c}\text { Sig. } \\
\text { (bilateral) }\end{array}$} \\
\hline & & \multirow{2}{*}{ Media } & \multirow{2}{*}{$\begin{array}{l}\text { Desviación } \\
\text { tip. }\end{array}$} & \multirow{2}{*}{$\begin{array}{l}\text { Error } \\
\text { tip. de la } \\
\text { media }\end{array}$} & \multicolumn{2}{|c|}{$\begin{array}{c}\text { 95\% Intervalo de } \\
\text { confianza para la } \\
\text { diferencia }\end{array}$} & & & \\
\hline & & & & & Inferior & Superior & & & \\
\hline Par 1 & $\begin{array}{l}\text { salto_M_DIR_post - } \\
\text { salto_M_DIR_pre }\end{array}$ & 2,273 & 11,909 & 2,073 & $-1,950$ & 6,496 & 1,096 & 32 & 0,281 \\
\hline Par 2 & $\begin{array}{l}\text { salto_RS_PROBL_post - } \\
\text { salto_RS_PROBL_pre }\end{array}$ & 5,241 & 10,763 & 1,999 & 1,148 & 9,335 & 2,623 & 28 & 0,014 \\
\hline
\end{tabular}

La Tabla 3 de prueba de muestras relacionadas del pre-test y el post-test, de la prueba de salto largo a pies juntos, correspondiente al estilo de enseñanza tradicional (Mando Directo) y el estilo de enseñanza alternativo (Resolución de problemas) proporcionado a cada grupo, se puede apreciar que existe diferencia estadísticamente significativa a un nivel de confianza del 95\% ( $\mathrm{p}<0,05)$ en la aplicación de la intervención de trabajo del aumento de los niveles de fuerza del tren inferior .
El estilo de enseñanza alternativo, ya sea Resolución de problemas, presenta diferencias estadísticamente significativas $(\mathrm{p}$-valor $=$ $0,014)$ en relación al test y post-test de la prueba de salto largo a pies juntos.

Respecto al estilo de enseñanza tradicional, correspondiente al Mando directo, presenta cambios, pero no son estadísticamente significativos por su valor $(\mathrm{p}$-valor $=0,281$ ). 
Tabla 4

Prueba de muestra independiente.

\begin{tabular}{|c|c|c|c|c|c|c|c|c|c|c|}
\hline & & \multicolumn{3}{|c|}{$\begin{array}{c}\text { Prueba de Levene } \\
\text { para la igualdad de } \\
\text { varianzas }\end{array}$} & \multicolumn{6}{|c|}{ Prueba T para la igualdad de medias } \\
\hline & & \multirow{2}{*}{$\mathrm{F}$} & \multirow{2}{*}{ Sig. } & \multirow{2}{*}{$t$} & \multirow{2}{*}{$\mathrm{gl}$} & \multirow{2}{*}{$\begin{array}{c}\text { Sig. } \\
\text { (bilateral) }\end{array}$} & \multirow{2}{*}{$\begin{array}{l}\text { Diferencia } \\
\text { de medias }\end{array}$} & \multirow{2}{*}{$\begin{array}{c}\text { Error } \\
\text { tip. de la } \\
\text { diferencia }\end{array}$} & \multicolumn{2}{|c|}{$\begin{array}{c}\text { 95\% Intervalo de } \\
\text { confianza para la } \\
\text { diferencia }\end{array}$} \\
\hline & & & & & & & & & Inferior & Superior \\
\hline \multirow[t]{2}{*}{ M_DIR_RS_PROBL_pre } & $\begin{array}{l}\text { Se han asumido } \\
\text { varianzas iguales }\end{array}$ & 2,671 & 0,107 & $-1,845$ & 60 & 0,070 & $-15,447$ & 8,373 & $-32,196$ & 1,302 \\
\hline & $\begin{array}{l}\text { No se han asumido } \\
\text { varianzas iguales }\end{array}$ & & & $-1,815$ & 52,512 & 0,075 & $-15,447$ & 8,512 & $-32,524$ & 1,629 \\
\hline \multirow[t]{2}{*}{ M_DIR_RS_PROBL_post } & $\begin{array}{l}\text { Se han asumido } \\
\text { varianzas iguales }\end{array}$ & 1,558 & 0,217 & $-2,041$ & 60 & 0,046 & $-18,416$ & 9,022 & $-36,463$ & $-0,368$ \\
\hline & $\begin{array}{l}\text { No se han asumido } \\
\text { varianzas iguales }\end{array}$ & & & $-2,014$ & 54,095 & 0,049 & $-18,416$ & 9,143 & $-36,745$ & $-0,087$ \\
\hline
\end{tabular}

La Tabla 4 de prueba de muestras independientes del test y el post-test, de la prueba de salto largo a pies juntos, correspondiente al estilo de enseñanza tradicional (Mando directo) y el estilo de enseñanza alternativo (Resolución de problemas) proporcionado a cada grupo, se expone que en el pre-test en ambos estilos de enseñanza (Mando directo - Resolución de problemas) existe diferencia estadísticamente significativa a un nivel de confianza del $95 \%$ $(\mathrm{p}<0,05)$ en la aplicación de la intervención de trabajo del aumento de los niveles de fuerza del tren inferior, en beneficio de la metodología de enseñanza de Resolución de problemas.

En la muestra independiente de pre-test de salto largo a pies juntos en comparación a ambos estilos de enseñanza, no presenta diferencias estadísticamente significativas por su valor $(\mathrm{p}$-valor $=0,070)$.

En la muestra independiente de post-test de salto largo a pies juntos en comparación a ambos estilos de enseñanza, se presentan diferencias estadísticamente significativas ( $\mathrm{p}$-valor $=0,046$ ).

Tabla 5

ANOVA de un factor.

\begin{tabular}{llccccc}
\hline & & $\begin{array}{c}\text { Suma de } \\
\text { cuadrados }\end{array}$ & gl & $\begin{array}{c}\text { Media } \\
\text { cuadrática }\end{array}$ & F & Sig. \\
\hline M_DIR_RS_PROBL_pre & Inter-grupos & 3683,168 & 1 & 3683,168 & 3,403 & 0,070 \\
\hline & Intra-grupos & 64930,268 & 60 & 1082,171 & & \\
\hline & Total & 68613,435 & 61 & & & \\
\hline M_DIR_RS_PROBL_post & Inter-grupos & 5234,863 & 1 & 5234 & 4,166 & 0,046 \\
\hline & Intra-grupos & 75390,104 & 60 & 1256,502 & & \\
\hline & Total & 80624,968 & 61 & & & \\
\hline
\end{tabular}

La Tabla 5 de prueba ANOVA de un factor, nos confirma que no se encuentran diferencias estadísticamente significativas en el pre-test entre ambos cursos para la prueba de salto largo a pie juntos (p- valor $=0,070$ ).
Para el post-test de salto largo a pies juntos entre ambos estilos de enseñanza, se presenta diferencias estadísticamente significativas en la prueba en cuestión, por su valor ( $\mathrm{p}$-valor $=0,046$ ), en un nivel de confianza del $95 \%$ 
$(\mathrm{p}<0,05)$, lo que indica que una estrategia, en este caso Resolución de Problemas, presenta mejoras en los procesos de aprendizaje, en comparación a la de Mando directo.

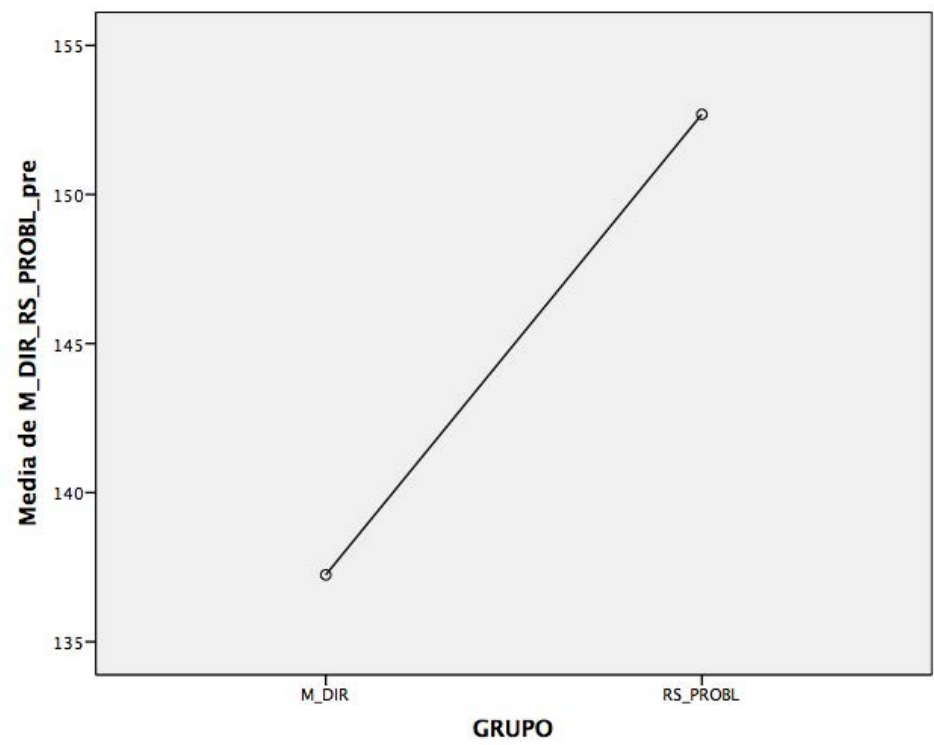

Figura 1. Media del pre-test de la prueba de salto largo a pies juntos, según estilo de enseñanza.

La Figura 1 muestra la media correspondiente a la prueba pre-test de salto largo a pies juntos, la cual arroja que no existen diferencias esta- dísticamente significativas entre ambos cursos en relación a los valores que arrojó la prueba de salto.

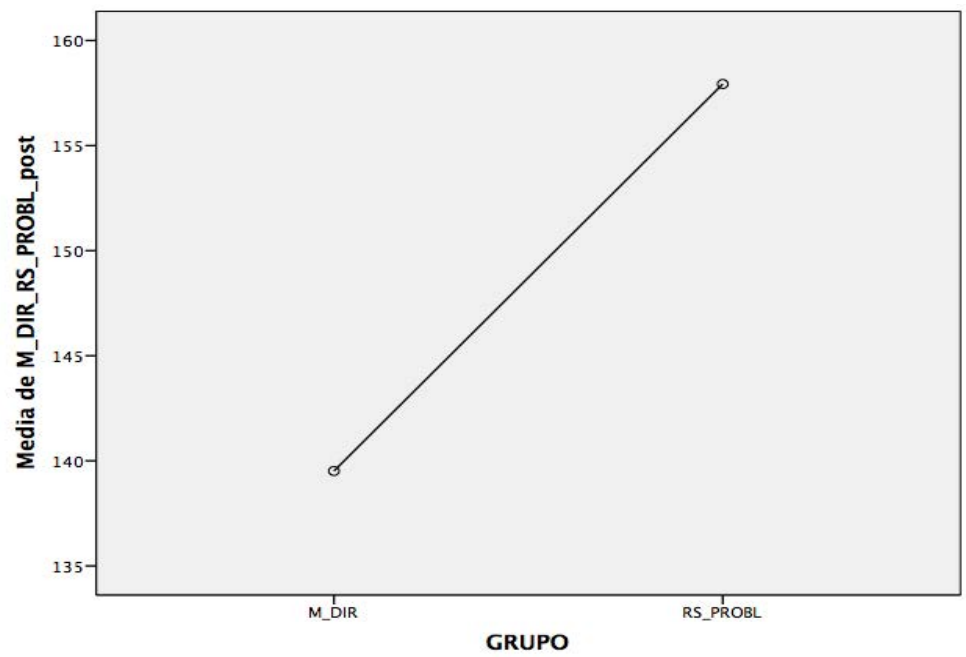

Figura 2. Media del post-test de la prueba de salto largo a pies juntos, según estilo de enseñanza. 
La Figura 2, muestra la media correspondiente a los estilos de enseñanza Mando directo y Resolución de problemas en la prueba post-test de salto largo a pies juntos, la cual arroja el efecto de un estilo sobre el otro (el efecto del estilo de enseñanza basado en el constructivismo es mayor que el de Mando directo) implica una diferencia estadísticamente significativa sobre el rendimiento del salto longitudinal pie juntos.

Tabla 6

Cálculo del tamaño del efecto.

\begin{tabular}{lcccccc}
\hline \multicolumn{7}{c}{ Pruebas de los efectos inter-sujetos } \\
\hline Variable dependiente: Salto_post & $\begin{array}{c}\text { Suma de cuadrados } \\
\text { tipo III }\end{array}$ & gl & $\begin{array}{c}\text { Media } \\
\text { cuadrática }\end{array}$ & F & Sig. & $\begin{array}{c}\text { Eta al } \\
\text { cuadrado parcial }\end{array}$ \\
\hline Origen & $5234,863^{\mathrm{a}}$ & 1 & 5234,863 & 4,166 & 0,046 & 0,065 \\
\hline Modelo corregido & 1365642,605 & 1 & 1365642,605 & 1086,861 & 0,000 & 0,948 \\
\hline Intersección & 5234,863 & 1 & 5234,863 & 4,166 & 0,046 & 0,065 \\
\hline estilo_enseñanza & 75390,104 & 60 & 1256,502 & & & \\
\hline Error & 1441042,000 & 62 & & & & \\
\hline Total & 80624,968 & 61 & & & & \\
\hline Total corregida & & & & & & \\
\hline
\end{tabular}

La Tabla 6 muestra la prueba para el calculo del efecto y en la que se puede observar que el nivel de significancia corresponde a un valor de 0,046 , lo que indica que los valores obtenidos en la post-prueba de salto longitudinal a pie juntos, demuestran que existen diferencias entre los grupos que utilizaron metodologías diferentes y que esa diferencia a favor de la metodología de Resolución de problemas genera mayor interés por parte del estudiante, redundando en un proceso de trabajo de mayor calidad por parte del estudiantado obteniendo estos resultados.

\section{Tabla 7}

Prueba para una muestra.

\begin{tabular}{ccccccc}
\hline & \multicolumn{9}{c}{ Valor de prueba $=0$} \\
\cline { 3 - 7 } & & & & & \\
& $\mathrm{t}$ & gl & Sig. (bilateral) & Diferencia de medias & $\begin{array}{c}\text { 95\% Intervalo de confianza } \\
\text { para la diferencia }\end{array}$ \\
\cline { 3 - 7 } & 2,530 & 61 & 0,014 & 3,66129 & 0,7680 & 6,5546 \\
\hline
\end{tabular}

La Tabla 7, presenta el análisis para la muestra, la cual calcula la diferencia entre el pre-test y el post-test de los dos grupos, el que arroja una diferencia estadísticamente significativa a un nivel de confianza del 95\% ( $\mathrm{p}<0,05)$, debido a su valor $\mathrm{p}(\mathrm{p}$-valor $=0,014)$. Lo que se puede traducir que el método de enseñanza orientado al constructivismo, genera mejores aprendizajes en los estudiantes. 


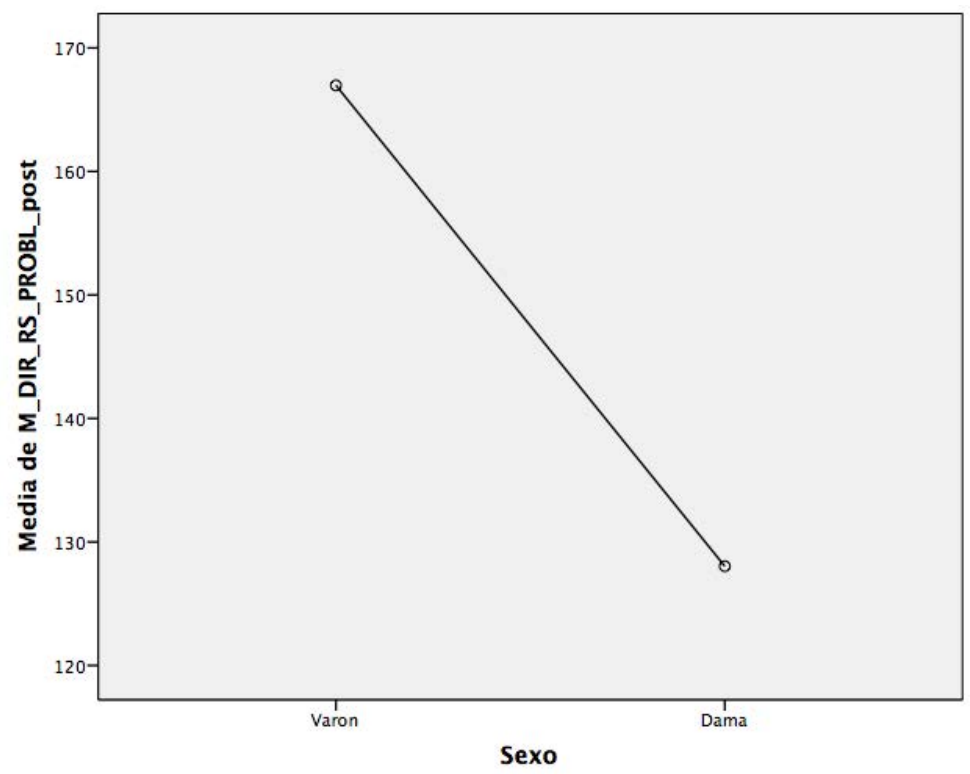

Figura 3. Media del post-test de la prueba de salto largo a pies juntos, según el sexo.

La Figura 3, muestra la media según el género correspondiente al post-test de la prueba de salto largo a pies juntos, la cual indica la existencia de diferencia estadísticamente significativa entre niños y niñas en el post-test, en cuanto a las distancias encontradas en el salto longitudinal a pie juntos.

\section{DISCUSIÓN}

Méndez (2005) \& Tallir et al. (2007) en sus estudios evalúan el impacto de ambas metodologías, donde concluyen que en los dos trabajos se obtienen mejores resultados utilizando una metodología alternativa: ya sea en pruebas relacionadas con la toma de decisiones o pruebas de técnica, No obstante, se requiere seguir indagando sobre cuál es la metodología de enseñanza más adecuada, métodos y medios más idóneos desde cada planteamiento, posibles influencias que podrían generarse como, el ámbito en el que se desarrolla la enseñanza, contexto, etapa formativa, etc.

Desde el modelo comprensivo (metodología alternativa), se crean propuestas que intervienen o modifican el juego real Thorpe, Bunker, \& Almond (1987) con el fin de que el aprendiz se implique cognitivamente en resolver la tarea Poveda \& Benítez (2010); Tallir et al. (2007) y así potenciar e incrementar el interés del deportista (Gray, Sproule, \& Morgan, 2009). La enseñanza del deporte desde el modelo comprensivo y utilizando una metodología constructivista, propone el uso de distintas situaciones de juego y organización a lo largo de la temporada, con la finalidad de beneficiar el aprendizaje del aprendiz (Cárdenas, 2006; Ibáñez, 2008; Oliveira \& Graça, 1997).

La metodología tradicional busca que el competidor domine las técnicas básicas del deporte, no así en el caso de la metodología alternativa donde su objetivo principal es el aprendizaje del competidor a lo largo del proceso (Méndez, 2005). Así lo puede demostrar el resultado obtenido por parte de la metodología de enseñanza vinculada al constructivismo (Resolución de problemas), en donde se observaron los mayores avances en relación al test inicial y al final en el salto longitudinal a pie juntos.

Garganta (1997) señala que emplear la metodología tradicional o mecanicista tiene gran importancia en el resultado del juego ya que la metodología es esencial en la fase de ejecu- 
ción. En contraposición con lo planteado por Ganrganta en 1997, los resultados que se obtuvieron arroja información que el desarrollo de los procesos cognitivos (Resolución de problemas) por parte de los alumnos generaron un mayor impacto en el logro de los objetivos de aprendizaje y los resultados en la prueba de salto longitudinal a pie junto así lo demuestran, concluyendo que el estilo de enseñanza derivado de una vertiente más constructivista puede influenciar y potenciar de mejor manera al estudiante que uno de la línea mas tradicional.

Para hablar de metodologías de enseñanza Mosston \& Ashworth (1986) hacen referencia que el término estilo de enseñanzas, métodos, aproximaciones, modelos y/o estrategias se empleaban y siguen aún empleándose de distintos modos por distintos autores. Habitualmente este término se refiere al estilo personal, pero el libro considera todo lo contrario, es decir, que el estilo de enseñanza hace referencia a una estructura independiente de las propias idiosincrasias. Razón por la cual en el MBE (2008), se plantea que la planificación de la enseñanza debe estar centrada en el alumno y conocer las características de ellos para obtener el máximo de rendimiento del estudiantado, lo que se condice con lo que puede buscar las estrategias constructivistas en las cuales el currículo no es el centro de proceso, sino que el eje esta dado en el aprendizaje del estudiante y es lo que se pretendió realizar en este estudio, ver como la estrategia de origen constructivista puede influir en el aprendizaje del estudiante, obteniendo resultados superiores comparados con un modelo tradicional de enseñanza.

\section{CONCLUSIÓN}

Los resultados obtenidos avalaron la hipótesis planteada en la investigación, la que dice que existen cambios estadísticamente significativos entre el estilo de enseñanza alternativo (Resolución de problemas) y el estilo de enseñanza tradicional (Mando directo) en la cualidad física de fuerza del tren inferior aplicados a la prueba de salto largo a pies juntos, en el colegio Mistral de la comuna Las Cabras.
Por otra parte una de las contribuciones importantes que deja este estudio; es el estilo de enseñanza que proporciona resultados estadísticamente significativos, los cuales son favorables en la prueba de salto largo a pies juntos, y que tiene una orientación alternativa, como la Resolución de problemas; esto se genera a través de un plan de entrenamiento de 6 semanas aproximadamente, la cual busca que el estudiante le dé una respuesta o solución al problema establecido por el docente, basándose en los planes y programas establecidos por el gobierno, dando un énfasis al desarrollo cognitivo del estudiante.

Dentro de las limitantes que tiene la investigación es la utilización de tan solo dos estilos de enseñanza, esto debido al poco tiempo y el poco personal humano para su realización, se pudo haber trabajado con más estilos de enseñanza el cual permite tener una visión más amplia de las diferentes formas de trabajo que puede adoptar un profesor frente a su clase, del mismo modo se puede agregar un mayor número de docentes comprometidos este tipo de estudio, además de otros establecimientos para aumentar la muestra de la investigación.

Otro aporte que entrega este trabajo, es el modo personal de cada docente al momento de realizar la clase de educación física, ya que nos encontramos con docentes los cuales ya tienen integrado o mayormente trabajaban con cierto tipo de estilo de enseñanza; esto conlleva a los alumnos a trabajar de cierto modo, ya que internalizan un cierto prototipo de aprendizaje frente a las clases; esto quiere decir, que los alumnos ya tienen un modo de trabajo; por lo cual un nuevo estilo de enseñanza conlleva a que el estudiante tenga un proceso de adaptación a lo nuevo que se le está entregando, en el proceso de enseñanza - aprendizaje.

Para futuros estudios seria de mucha importancia variar con la mayoría de los estilos de enseñanza, como abanico de posibilidades frente a las clases de educación física o una prueba específica; así también como generar momentos en las cuales los alumnos opinen, acerca del estilo que se le hizo más favorable o cómodo al momento del proceso enseñanzaaprendizaje. 


\section{REFERENCIAS BIBLIOGRÁFICAS}

Almond, L. (1987). Rethinking athletics. Physical Education Review, 10(1), 17-20.

Aguirre de Vergara, M. (2011). El docente como facilitador y mediador. (IV Congreso de Calidad Educativa). Quito: Universidad de Quito.

Cárdenas, D. (2006). El proceso de formación táctica colectiva en el baloncesto desde la perspectiva constructivista. Lecturas Educación Física y Deportes, Revista Digital, 94. Reviewed August 1, 2009, at http://www.efdeportes.com/efd94/ balonces.htm

Echeverria, R. (2014). La resolución de problemas como estrategia de enseñanza para el aprendizaje de la táctica ofensiva en la categoría pre-benjamín de fútbol 8 . Revista psicológica del deporte, 23,209.

Educarchile (2006) El constructivismo pedagógico. Recuperado el 24 de abril del 2017 de: www.educarchile.cl/UserFiles/ P0001/File

Delgado, M. \& Sicilia, A. (2002). Educación Física y Estilos de enseñanza. España: INDE.

Hernández, R., Fernández, C. y Baptista, P. (2006). Metodología de la investigación, sexta edición. México: Mc Graw Hill.

García, A., (1994). Didáctica e innovación curricular. Sevilla: Secretariado de Publicaciones de la Universidad de Sevilla.

Garganta, J. (1997). La enseñanza de los juegos deportivos. Barcelona: Paidotribo

Gray, S., Sproule, J. \& Morgan, K. (2009). 'Teaching Team Invasion Games and Motivational Climate'. European Physical Education Review, vol 15, no. 1, pp. 6589. DOI: $10.1177 / 1356336 \mathrm{X} 09105212$

Ibarra, L. (2006). El Rol del Profesor. La Habana: Universidad de la Habana.
MBE. (2008). Marco para la buena enseñanza. Recuperado el 20 de abril del 2017 de www.docentemas.cl/docs/MBE2008. $\underline{\mathrm{pdf}}$

Méndez, A. (2005). Técnicas de enseñanza en la iniciación al baloncesto. Zaragoza: Inde.

Méndez Giménez, A. (2005). Hacia una evaluación de los aprendizajes consecuente con los modelos alternativosde iniciación deportiva. Tándem, Didáctica de la Educación Física, 17, 38-58.

Mosston, M., Penhos, J., Cratty, B. J. \& Lederman, S. (1982). La enseñanza de la Educación Física. España: Hispanoamericana

Oliveira, J. \& Graça, A. (1997). La enseñanza del baloncesto. In Graça, A. y Oliveira, J. La enseñanza de los juegos deportivos, (pp.61-96). Barcelona: Paidotribo.

Poveda, J., Benítez, J. (2010). Fundamentos teóricos y aplicación práctica de la toma de decisiones en el deporte. E-Bm.com. Revista de Ciencias del Deporte, 6(2), 101-110.

Riverón, F., \& López Y. (2015). Influencia de los medios alternativos en las clases de Educación Física. Cuba: Universidad de Matanzas Camilo Cienfuegos.

\section{Dirección para correspondencia}

José Andrés Duarte Cornejo

Profesor de Educación Física

Licenciado en Educación

Magíster en Educación Física con mención en

Actividad Física y Salud

Universidad Católica del Maule, Chile.

Antropometrista ISAK nivel 3.

Docente Universidad Autónoma de Chile.

Contacto:

jose_d81@hotmail.com

Recibido: 20-10-2017

Aceptado: 26-11-2017 